\title{
Vibrations of a geometrically nonlinear viscoelastic shallow shell with concentrated masses
}

\author{
Nikolai Vatin ${ }^{1}$, Rustamkhan Abdikarimov ${ }^{2}$ and Dadakhan Khodzhaev ${ }^{3^{*}}$ \\ ${ }^{1}$ Peter the Great St.Petersburg Polytechnic University, St.Petersburg, Russia \\ ${ }^{2}$ Tashkent Institute of Finance, Tashkent, Uzbekistan \\ ${ }^{3}$ Tashkent Institute of Irrigation and Agricultural Mechanization Engineers, Tashkent, Uzbekistan
}

\begin{abstract}
Shell structures are widely used in various fields of technology and construction. Often, they play the role of a bearing surface with assemblies, overlays, and aggregates installed on them. At the same time, in solving various problems, such attached elements are considered as the elements concentrated at the points and rigidly connected. Vibrations of an orthotropic viscoelastic shallow shell with concentrated masses in a geometrically nonlinear setting are considered. The equation of motion for a shallow shell is derived based on the Kirchhoff-Love theory. The traditional Boltzmann-Volterra theory is used to describe the viscoelastic properties of a shallow shell. The effect of concentrated masses is taken into account using the Dirac delta function. Using the polynomial approximation of the deflections of the Bubnov-Galerkin method, the problem is reduced to solving a system of ordinary nonlinear integrodifferential equations with variable coefficients. In the calculations, the three-parameter Koltunov-Rzhanitsyn kernel was used as a weakly singular relaxation kernel. A numerical method was used to solve the resulting system that eliminates the singularity in the relaxation kernel. The problem of nonlinear vibrations of an orthotropic viscoelastic shallow shell with concentrated masses is solved. The influence of concentrated masses and location, properties of the shell material, and other parameters on the amplitude-frequency response of the shallow shell vibrations is investigated.
\end{abstract}

\section{Introduction}

Shell structures are widely used in many areas of the industry: in hydraulic engineering, in construction, in various agricultural and industrial facilities. An important aspect is the reduction of energy and material consumption. One solution to this problem is the use of composite materials that most fully meet the features and operating conditions of structures. Often, such structures play the role of a bearing surface on which various assemblies,

\footnotetext{
*Corresponding author: dhodjaev@mail.ru
} 
engines, devices, equipment, and the transported cargo are attached. In calculations, such additional elements are considered to be concentrated at the points and rigidly connected.

The study in $[1,2]$ is devoted to the numerical and experimental investigation of nonlinear vibrations of a rectangular plate carrying various concentrated masses. For a numerical study, a Karman-type equation is solved, the results of which are compared with the experimental one.

In [3], the vibrations of elastic plates with concentrated masses are investigated. The results obtained analytically are compared with the results obtained using the finite element method (FEM).

[4] is devoted to the study of vibrations of plates with concentrated masses. Numerical results are obtained using ANSYS software. The influence of the number of concentrated masses and their location on the eigenfrequencies of the plate vibrations was studied.

The dynamics of sheet metal with concentrated masses were considered in [5]. The influence of the concentrated mass on the eigenfrequency of vibrations of sheet metal was investigated.

In [6], the frequency and modes of vibrations of a thin shell with an associated mass are investigated. The solution to the equation of motion was obtained by the Bubnov-Galerkin method.

In [7], the stability of a hinged rectangular plate carrying an arbitrary associated mass is considered. Using the Galerkin method, an equation of the Karman type is solved. The influence of the concentrated mass and its location on the amplitude-frequency response of the plate is investigated.

Vibrations of thin plates with concentrated masses are considered in [8]. Analytical solutions of the problem are compared with the results obtained by the finite element method.

In [9], free vibrations of composite plates and panels with a mass concentrated in the center are investigated under various boundary conditions. The solution to the problem is obtained by the finite element method.

In [10], parametric vibrations of a thin rectangular plate with a concentrated mass are considered. The solution to the problem was obtained by the Bubnov-Galerkin method.

Along with this, it is worth mentioning the studies devoted to the problems of the dynamics of elastic and viscoelastic plates and shells with concentrated masses [11-13].

A review of the literature sources shows that insufficient attention was paid to the study of nonlinear vibrations and dynamic stability of plates, panels, and shells with concentrated masses, taking into account the viscoelastic and inhomogeneous properties of the material [14-17].

The paper investigates the vibrations of an orthotropic viscoelastic shallow shell with concentrated masses, taking into account geometric nonlinearity.

\section{Material and methods}

Consider an orthotropic viscoelastic rectangular shallow shell with dimensions $a \times b$ and with radii of curvature of the middle surface $R_{1}$ and $R_{2}$, carrying concentrated masses $M_{i}$ at the points with coordinates $\left(x_{i}, y_{i}\right)$.

The effect of concentrated masses is introduced into the equation of motion using the Dirac delta function [18].

A weakly singular Koltunov-Rzhanitsyn kernel of the following form [19] is used as a relaxation kernel.

$$
\Gamma(t)=A e^{-\beta t} \cdot t^{\alpha-1}, \quad A>0, \quad \beta>0, \quad 0<\alpha<1 .
$$


Under the accepted assumptions, the equation of motion of an orthotropic viscoelastic shallow shell with concentrated masses relative to deflection $w=w(x, y, t)$ and displacements $u=u(x, y, t), v=v(x, y, t)$ is described by the system of integro-differential equations.

In the general case, the boundary conditions can be of any kind. By the BubnovGalerkin method, we represent functions $w(x, y, t), u(x, y, t), v(x, y, t)$ in the form of an expansion in functions $\phi_{n m}(x, y), \varphi_{n m}(x, y), \psi_{n m}(x, y)$, satisfying corresponding boundary conditions:

$$
\begin{gathered}
u(x, y, t)=\sum_{n=1}^{N} \sum_{m=1}^{M} u_{n m}(t) \phi_{n m}(x, y), v(x, y, t)=\sum_{n=1}^{N} \sum_{m=1}^{M} v_{n m}(t) \varphi_{n m}(x, y) \\
w(x, y, t)=\sum_{n=1}^{N} \sum_{m=1}^{M} w_{n m}(t) \psi_{n m}(x, y)
\end{gathered}
$$

where $u_{n m}=u_{n m}(t), v_{n m}=v_{n m}(t), w_{n m}=w_{n m}(t)$ are the unknown functions of time; $\phi_{n m}(x, y), \quad \varphi_{n m}(x, y), \quad \psi_{n m}(x, y), \quad n=1,2, \ldots, N ; \quad m=1,2, \ldots, M \quad$ are the coordinate functions that meet the given boundary conditions of the problem.

Substituting (1) into the equation of motion and applying the Bubnov-Galerkin method to determine the unknowns $u_{n m}(t), v_{n m}(t), w_{n m}(t)$, we obtain a nonlinear system of integro-differential equations with variable coefficients.

Further, integrating the obtained values twice over $t$, we write it in integral form. Using the numerical method proposed in [20], assuming that $t=t_{i}, t_{i}=i \Delta t, i=1,2, \ldots$ ( $\Delta t$ is the integration step) and replacing the integrals with quadrature trapezoidal formulas to calculate the unknowns $w_{\text {inm }}=w_{\text {inm }}\left(t_{i}\right), u_{\text {inm }}=u_{\text {inm }}\left(t_{i}\right), v_{\text {inm }}=v_{\text {inm }}\left(t_{i}\right)$, we obtain a recurrent formula.

\section{Results and Discussion}

Based on the developed computational algorithm, a program was created in the Delphi programming environment. This program allows us to study the amplitude-frequency response and the stress-strain state of a shallow shell. The results are summarized in the graphs below.

Figure 1 shows the time dependence of the deflection of the midpoint of elastic and viscoelastic shallow shells with concentrated masses. As expected, an account for viscoelastic properties of the shallow shell material leads to damping of the oscillatory process. 


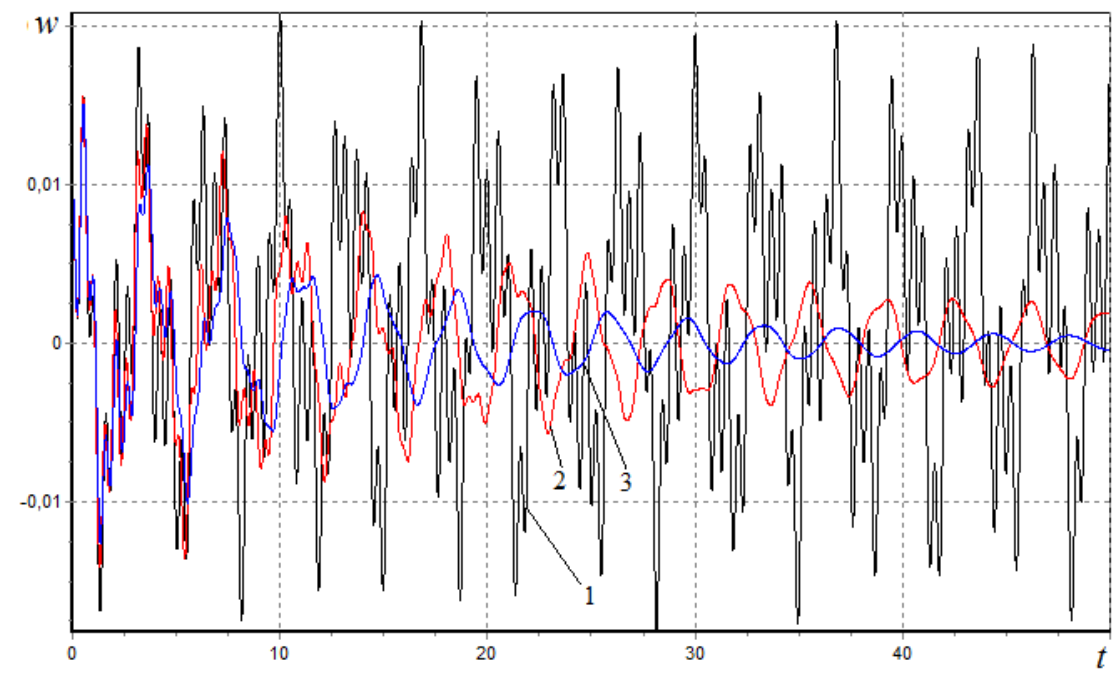

Fig. 1. Deflection versus time: $A_{i j}=0$ (1); 0.025 (2); 0.05 (3)

Figure 2 shows the vibration modes of an orthotropic elastic and viscoelastic shallow shell with concentrated masses.
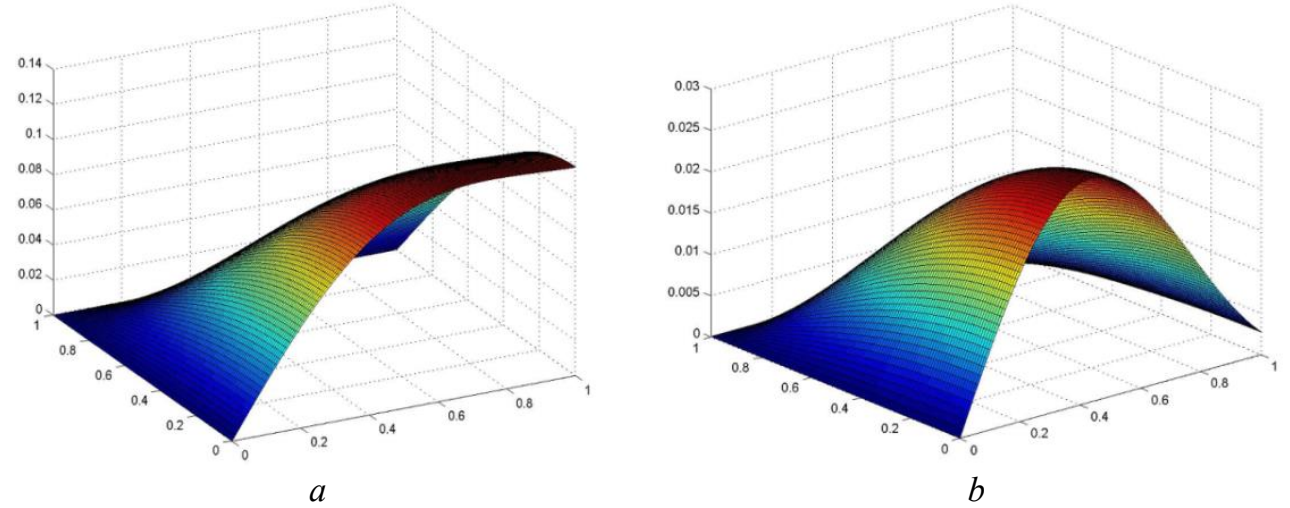

Fig. 2. Vibration modes of a shallow shell with concentrated masses at $t=12$ : a) elastic shell; b) viscoelastic shell

The influence of the concentrated mass on the oscillatory process is shown in Figure 3. It is seen that an increase in the concentrated mass leads to a decrease in the vibration amplitude. 


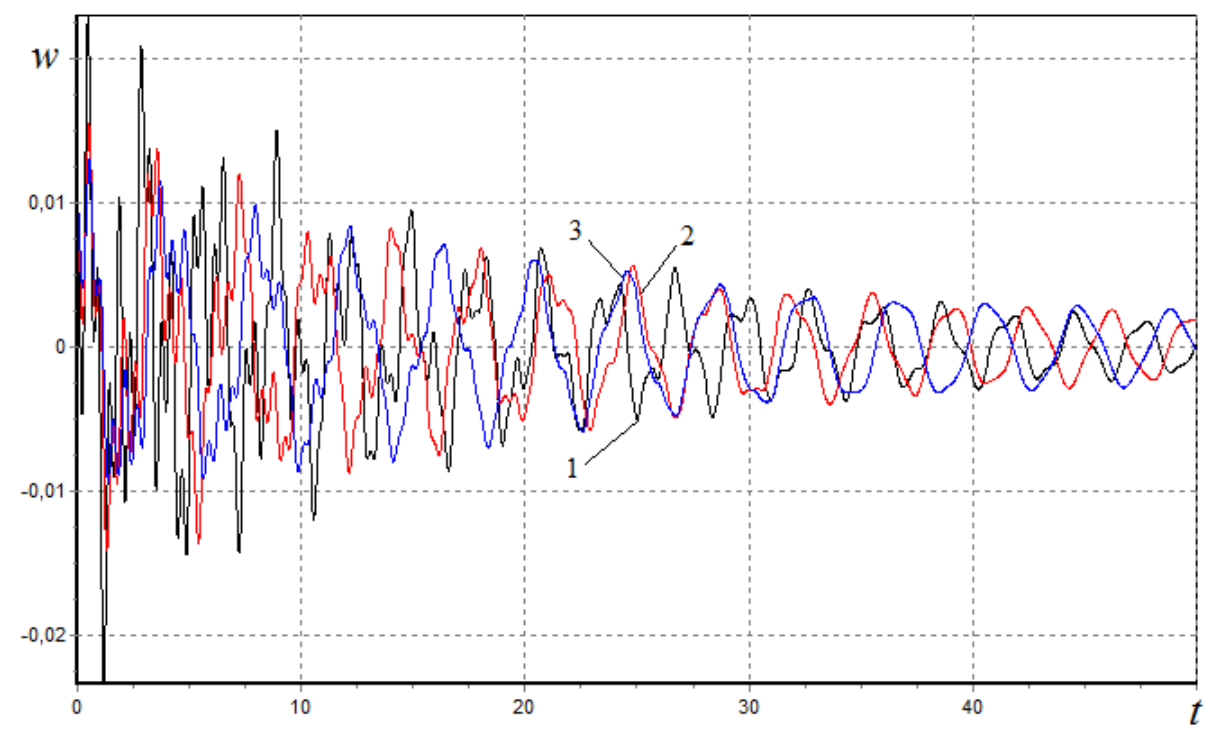

Fig. 3. Deflection versus time: 1) $M_{1}=0$

2) $M_{1}=0.1 \quad$ 3) $M_{1}=0.2$

Figure 4 shows the vibration modes of an orthotropic viscoelastic shallow shell with and without an account for the concentrated mass.

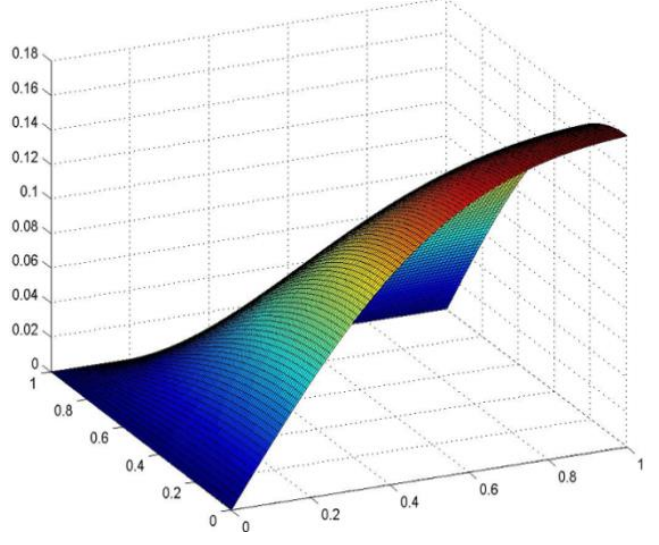

a)

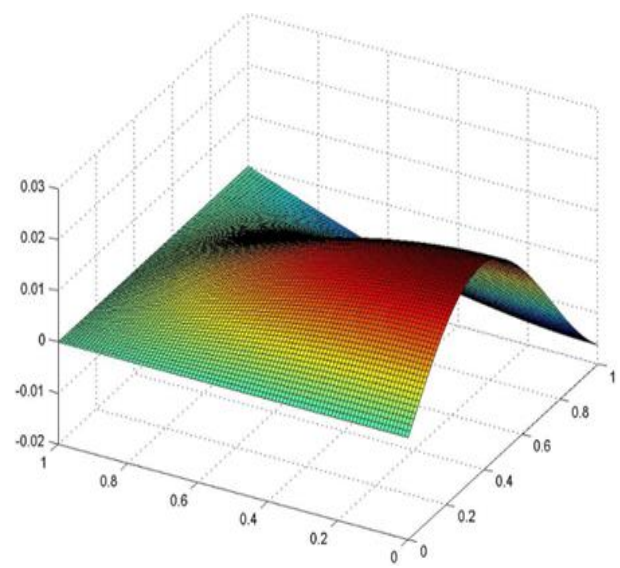

b)

Fig. 4. Vibration modes of a shallow shell at $t=12$ : a) without an account for the concentrated mass; $\mathrm{b}$ ) with an account for the concentrated mass

The results of studying the effect of an external static load $q$ on the behavior of a shallow shell with concentrated masses are shown in Figure 5. 


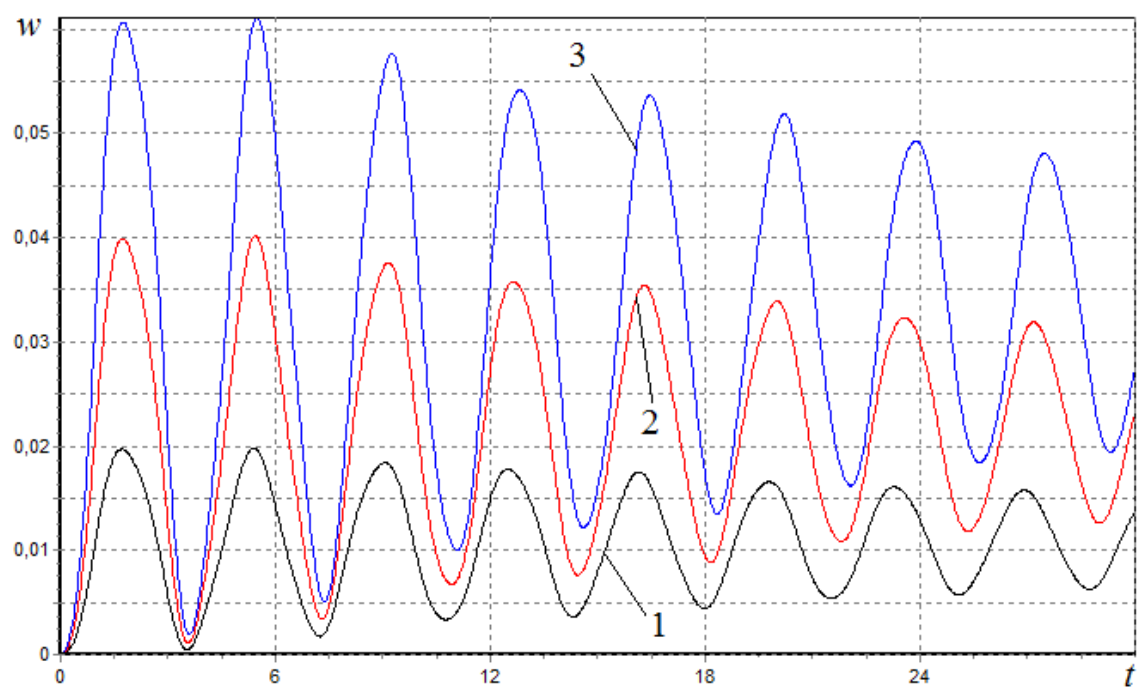

Fig. 5. Deflection versus time: $q=1$ (1), 2 (2), 3 (3)

The results obtained show that when the value of $q$ changes, the frequency of vibration does not change, while, with an increase in the static transverse load, the amplitude increases proportionally.

\section{Conclusion}

Vibrations of an orthotropic viscoelastic shallow shell with concentrated masses are investigated in a geometrically nonlinear formulation.

A mathematical model of the problem was constructed, an algorithm was developed, and a program was created to determine the deflection of a shallow shell carrying concentrated masses under various conditions of contour fixation.

The influence of changes in physical-mechanical and geometric parameters of the material of a shallow shell on the amplitude-time characteristics, as well as the parameters of concentrated masses and their location, were estimated.

\section{Funding}

This work is supported by the Russian Science Foundation under grant 21-19-00324.

\section{References}

1. M. Amabili, J. Sound Vib. 329, 4501 (2010)

2. M. Amabili and S. Carra, J. Sound Vib. 331, 155 (2012)

3. H. Qi, Z. Zhang, K. Chen, and F. Du, Eng. Mech. 32, 22 (2015)

4. N. Hossain, M. S. Islam, K. H. N. Ahshan, and M. Z. Hossain, J. Vibroengineering 17, $3751(2015)$

5. H. Zhao, G. Wang, and Y. Chen, in Proc. 2nd Int. Conf. Mechatronics Eng. Inf. Technol. (ICMEIT 2017) (Atlantis Press, n.d.), pp. 583-586

6. G. S. Leizerovich and S. V. Seregin, J. Appl. Mech. Tech. Phys. 57, 841 (2016)

7. Z. Zhong, A. Liu, Y. L. Pi, J. Deng, H. Lu, and S. Li, Eng. Struct. 196, 109288 (2019)

8. P. A. Martin and A. J. Hull, Wave Motion 98, (2020) 
9. A. Mandal, C. Ray, and S. Haldar, in Recent Adv. Theor. Applied, Comput. Exp. Mech. Lect. Notes Mech. Eng. (2020), pp. 49-57

10. Z.-L. Zhong and A.-R. Liu, Gongcheng Lixue/Engineering Mech. 37, 6 (2020)

11. T. Kocatürk, S. Sezer, and C. Demir, J. Sound Vib. 278, 789 (2004)

12. Y. Zhang, J. Vib. Acoust. Trans. ASME 133, (2011)

13. A. K. Chaubey, A. Kumar, and A. Chakrabarti, AIAA J. 56, 1662 (2018)

14. D. Khodzhaev, R. Abdikarimov, and N. Vatin, in MATEC Web Conf. (2018)

15. D. Khodzhaev, in E3S Web Conf. (2019)

16. M. M. Mirsaidov, R. A. Abdikarimov, and D. A. Khodzhaev, PNRPU Mech. Bull. 2019, 143 (2019)

17. D. Khodzhaev, R. Abdikarimov, and B. Normuminov, 896, (2020)

18. C. L. Amba-Rao, J. Appl. Mech. 31, 550 (1964)

19. L. E. Mal'tsev, Mech. Compos. Mater. 15, 131 (1979)

20. R. A. Abdikarimov and D. A. Khodzhaev, Mag. Civ. Eng. 49, 83 (2014) 